Slavica

bruxellensia

\section{Slavica bruxellensia}

Revue polyphonique de littérature, culture et histoire

slaves

9 | 2013

Érotisme

\title{
Bielecki M., Kłopoty z InnościĄ (Les Problèmes de l'Altérité)
}

\section{Natalia Gendaj}

\section{OpenEdition \\ Journals}

Édition électronique

URL : http://journals.openedition.org/slavica/1328

DOI : 10.4000/slavica.1328

ISSN : 2034-6395

Éditeur

Université libre de Bruxelles - ULB

\section{Référence électronique}

Natalia Gendaj, « Bielecki M., Kłopoty z Innością (Les Problèmes de l'Altérité) », Slavica bruxellensia [En ligne], 9 | 2013, mis en ligne le 15 avril 2013, consulté le 22 septembre 2020. URL : http:// journals.openedition.org/slavica/1328; DOI : https://doi.org/10.4000/slavica.1328

Ce document a été généré automatiquement le 22 septembre 2020.

\section{(c) $(1) \odot$}

Les contenus de Slavica bruxellensia sont mis à disposition selon les termes de la Licence Creative Commons Attribution - Pas d'Utilisation Commerciale - Pas de Modification 3.0 France. 


\title{
Bielecki M., Kłopoty z InnościĄ (Les Problèmes de l'Altérité)
}

\author{
Natalia Gendaj
}

\section{RÉFÉRENCE}

Bielecki M., Kłopoty z InnościĄ, Universitas, Cracovie, 2012, 294 p. 
1 La question de l'Autre ou plus généralement de l'Altérité se trouve de plus en plus souvent au centre de l'attention des chercheurs en littérature et en études culturelles. La popularité de cette problématique donne lieu à des travaux interdisciplinaires à la frontière des études littéraires, de l'anthropologie, de l'histoire, etc. L'Autre constitue également le principal objet d'analyse du nouveau livre de Marian Bielecki, livre au titre évocateur: Kłopoty z Innością (Les Problèmes de l'Altérité). Le chercheur de Wrocław étudie l'Autre par le biais de méthodologies qui traitent par essence de la catégorie de l'Autre et il se sert de leurs résultats, reprenant des concepts tels que ceux de l'étrangeté, de l'incompréhension ou de l'inégalité. Parmi les théories utilisées se trouvent donc: les études

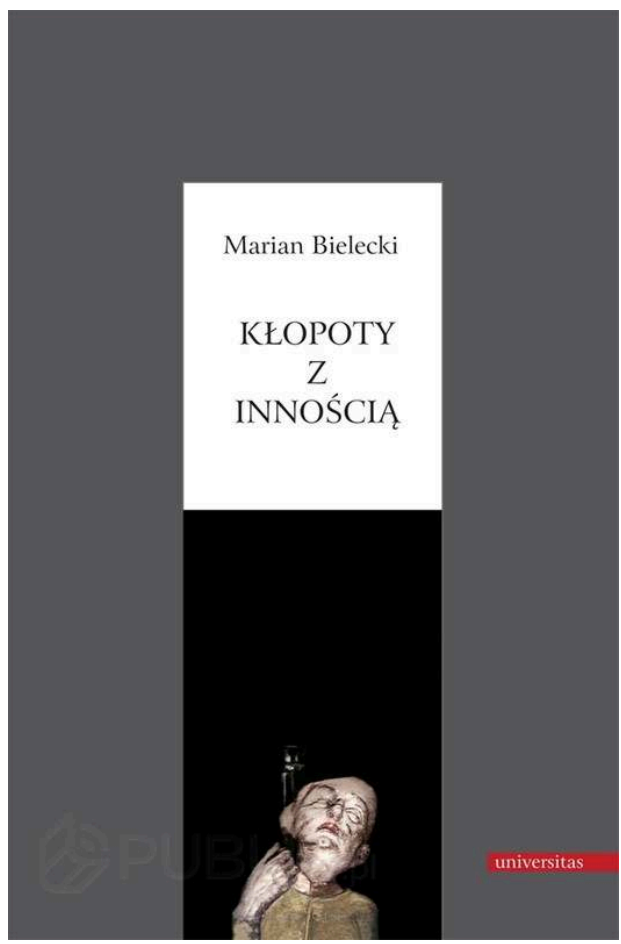
postcoloniales, la queer theory et la critique féministe.

2 Cette problématique est tellement bien ancrée dans la critique littéraire polonaise qu'il ne paraît pas nécessaire de multiplier les travaux basés sur des matrices méthodologiques qui sont devenues des lieux communs. Ce n'est pas la première fois qu'apparaissent les noms de Julia Kristeva (avec la catégorie de l'abjection), Roland Barthes (avec la subjectivité de l'auteur et du lecteur) et Susan Sontag (avec la sensibilité «camp »). En réalité, l'ouvrage de Bielecki est un recueil d'articles qui ont été publiés entre 2005 et 2012 dans plusieurs revues d'études littéraires (PamiĘtnik Literacki et Teksty Drugie) et culturelles (FA-ART-art et Pogranicze, cette dernière n'existant déjà plus). Ce n'est donc pas tant la lecture de textes individuels que la lecture du recueil dans son ensemble qui semble intéressante dans ce cas-ci.

$3 \quad$ Kłopoty $z$ InnościĄ est divisé en quatre parties, qui, toutefois, tournent toutes plus ou moins autour de la figure de Witold Gombrowicz. Les deux premières parties - Inność (Altérité) et Kamp (Camp) - ont un caractère plutôt interprétatif, alors que les deux dernières - Inne pisania (D'autres écritures) et Inne czytania (D'autres lectures) concernent (disons-le franchement) la personne en soi du chercheur en littérature. L'Autre chez Gombrowicz se compose de plusieurs tableaux et aborde différents aspects culturels. Il ou plutôt elle est incarnée par Yvonne (Yvonne, princesse de Bourgogne), laquelle perturbe les rôles et les schémas culturels établis. Gonzalo (Trans-Atlantique), qui se caractérise par une sensibilité, une esthétique ( camp ») et une sexualité (queer studies) particulières, est également l'Autre. Enfin, pour Gombrowicz-reporter, les Argentins sont, eux aussi, des Autres (Pérégrinations argentines). Ce dernier aspect est tellement intéressant qu'il continue d'être discuté en Pologne. L'ensemble de ses reportages pour la Radio Free Europe a été négligé tant par leur auteur («j'écris des petits feuilletons innocents, un pur gagne-pain $\left.»^{1}\right)$ que par la critique polonaise; d'ailleurs, ses réflexions concernant le Nouveau Continent n'étaient pas toujours 
originales, et n'étaient certainement pas tout à fait les siennes: Gombrowicz s'est souvent nourri des théories de l'extravagant philosophe Herman Keyserling, bien que Bielecki n'en dise rien². L'auteur de Kłopoty z Innością base souvent ses interprétations sur les travaux des spécialistes étrangers de Gombrowicz (principalement de l'autre côté de l'Atlantique), ne se référant que de temps en temps au Czarny nurt (Le courant noir) de Michał Paweł Markowski. Jusqu'ici, les recherches sur Gombrowicz en Pologne étaient à peu près monopolisées par les chercheurs nationaux, Jerzy Jarzębski en tête. Chez Bielecki, les chercheurs polonais sont présents, mais ils jouent un tout autre rôle. C'est à eux, entre autres, que sont consacrées les deux parties restantes du livre.

4 Konstanty Jeleński et Jerzy JarzĘbski, le propagateur de l'œuvre de Gombrowicz et son principal interprétateur, sont deux figures qui intéressent Bielecki. Il ne s'agit pas ici d'utiliser leur acquis scientifique, mais il est question de leur manière de travailler, de leur relation avec les textes de Gombrowicz. Pour Bielecki, Jeleński est un chercheur en littérature qui se base sur un discours académique à l'excès. Sa méthodologie est le manque de méthodologie. En revanche, il estime JarzĘbski pour sa close reading personnelle (si l'on s'en tient aux problèmes de théorie littéraire). Le spécialiste cracovien de Gombrowicz lit avant tout les textes attentivement, et puis seulement bien que rarement - il les applique à une théorie. C'est sans doute de là que vient l'approche parfois trop autoritaire de JarzĘbski vis-à-vis des travaux scientifiques des autres chercheurs.

5 Les textes consacrés aux deux spécialistes ne sont pas seulement un simple supplément au livre sur Gombrowicz. Ces deux noms jouent un rôle important dans Kłopoty $z$ Innością: ils sont les Autres successifs, c'est-à-dire des lecteurs qui se distinguent clairement du reste. Les essais consacrés à Jarzębski et à Jeleński, bien que placés à la fin de l'ouvrage, peuvent être une forme d'explication au style de l'auteur et en même temps son hypothèse programmatique. Bielecki signale clairement sa subjectivité dans le texte scientifique, souvent il exprime des opinions personnelles, relevant plus du feuilleton que du discours académique.

\section{NOTES}

1. Lettre de Gombrowicz à Artur Sandauer du 27 novembre 1959, Gombrowicz. Walka o sławĘ. Korespondencja Witolda Gombrowicza z Józefem Wittlinem, Jarosławem Iwaszkiewicz, Arturem Sandauerem (La Lutte pour la célébrité. Correspondance de Witold Gombrowicz avec Józef Wittlin, Jarosław Iwaszkiewicz et Artur Sandauer), Jarz̨̨bski J. (éd.), Wydawnictwo Literackie, Cracovie, 1996, p. 235.

2. Aleksander Fiut en parle dans son article«Zwiedzanie Kontynentu z "Trzeciego Dnia Stworzenia": Gombrowicz i Keyserling " (La visite du Continent du "Troisième Jour de la Création »: Gombrowicz et Keyserling), in : Ruch Literacki, n², 2005, pp. 167-181. 


\section{AUTEURS}

NATALIA GENDAJ

Université de Szczecin 